Rabaska

Revue d'ethnologie de l'Amérique française

\title{
Jean-Michel Guilcher (1914-2017)
}

\section{Fañch Postic}

Volume 15, 2017

URI : https://id.erudit.org/iderudit/1041130ar

DOI : https://doi.org/10.7202/1041130ar

Aller au sommaire du numéro

Éditeur(s)

Société québécoise d'ethnologie

ISSN

1703-7433 (imprimé)

1916-7350 (numérique)

Découvrir la revue

Citer ce document

Postic, F. (2017). Jean-Michel Guilcher (1914-2017). Rabaska, 15, 199-203.

https://doi.org/10.7202/1041130ar

Ce document est protégé par la loi sur le droit d'auteur. L'utilisation des services d'Érudit (y compris la reproduction) est assujettie à sa politique d'utilisation que vous pouvez consulter en ligne.

https://apropos.erudit.org/fr/usagers/politique-dutilisation/
Cet article est diffusé et préservé par Érudit.

Érudit est un consortium interuniversitaire sans but lucratif composé de l'Université de Montréal, l'Université Laval et l'Université du Québec à Montréal. Il a pour mission la promotion et la valorisation de la recherche. https://www.erudit.org/fr/ 


\section{Nécrologie}

\section{Jean-Michel Guilcher (1914-2017) ${ }^{1}$}

L'ethnologue Jean-Michel Guilcher nous a quittés le 24 mars 2017, discrètement, à l'image finalement de ce que fut sa longue vie. Né en 1914 à Brest où sa mère était institutrice, il n'était en effet pas du genre à se mettre sur le devant de la scène. Cela lui aurait peut-être valu plus de considération de la part de certains milieux de la recherche et de la culture qui ne l'ont pas toujours ménagé. Il n'en avait cure, considérant qu'on devait le juger sur son seul travail et qu'il n'avait ni à se plier aux modes du temps ni à solliciter une quelconque reconnaissance en se livrant à des activités de salon. Il est resté toute sa vie

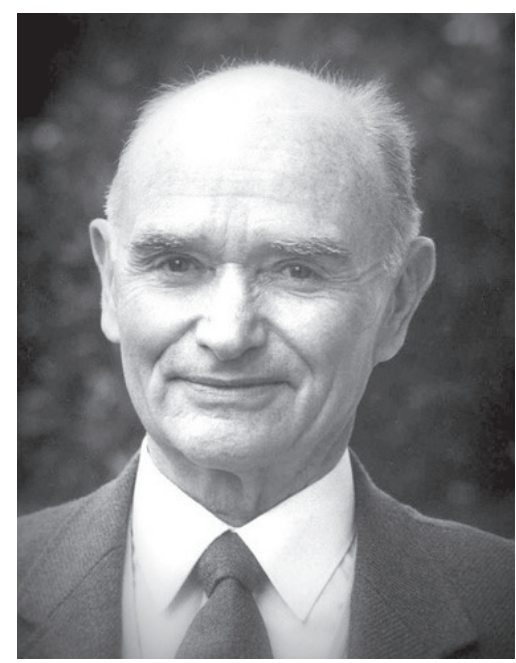

Photo : Philippe-Étienne Raviart, date inconnue un homme libre de ses mouvements et de ses pensées.

Après des études secondaires au lycée de Brest, Jean-Michel Guilcher s'inscrit à la Sorbonne où il prépare l'agrégation d'histoire naturelle. Il s'intéresse également et participe au mouvement d'éducation populaire que favorisent les premiers congés payés institués par le Front populaire. Hélène, celle qui deviendra son épouse - et son active collaboratrice tout au long

1. Cette notice reprend pour une part la communication proposée par Jean-François Simon et moi-même lors du colloque autour de l'œuvre de Marius Barbeau organisé à Saint-Irénée en octobre 2014, publiée sous le titre « Du folklore à l'ethnologie : "traditions populaires" et projet universitaire en Bretagne. La contribution fondamentale de Jean-Michel Guilcher », dans Présence de Marius Barbeau : l'invention du terrain en Amérique française. Autour d'un legs centenaire (1914-2014), sous la direction de Jean-Pierre Pichette, avec la collaboration de Serge Gauthier, Christian Harvey, Bertrand Bergeron et Jean Simard, Rabaska, vol. 13, 2015, p. 249-262. 
de ses recherches -, l'entraîne dans les cours de Miss Maud Pledge (18931949); cette dernière applique des méthodes pédagogiques originales à une gymnastique qui intègre le jeu et la danse. Tandis que la déclaration de guerre interrompt ses études, le repli en zone libre donne à Jean-Michel Guilcher l'opportunité de premières enquêtes sur la danse dans les Pyrénées ou en Berry au sein du mouvement Jeune France. Il publie même, dans la revue Esprit, un premier article sur la danse populaire où il reprend une théorie de la survivance alors largement acceptée par les milieux scientifiques. Cela lui vaut d'être vivement critiqué par Henri Davenson (pseudonyme d'Henri-Irénée Marou, 1904-1977). C'est l'occasion d'une autocritique qui entraîne une remise en question et des interrogations sur les mécanismes d'apprentissage et de transmission des danses traditionnelles. Pour y répondre, Jean-Michel Guilcher comprend qu'il conviendrait de reprendre la recherche sur des bases entièrement nouvelles et que cela nécessiterait une fine enquête de terrain pour laquelle - il en est persuadé - sa Bretagne natale offrirait le terrain idéal. Mais la guerre ne le permet pas...

En 1942, après la dissolution du mouvement Jeune France, Jean-Michel Guilcher entre dans la maison d'édition du Père Castor fondée en 1931 par Paul Faucher (1898-1967), promoteur de pédagogies nouvelles en matière d'éducation. Commence alors entre les deux hommes une étroite coopération que nourrit une réflexion sur les outils pédagogiques du futur et qui aboutira en 1945 à la création des «Ateliers du père Castor » auxquels Jean-Michel Guilcher collaborera activement pendant dix années.

Dès la Libération, Jean-Michel Guilcher et sa femme Hélène engagent des enquêtes sur la côte nord du Finistère. C'est aussi à cette époque qu'il fait la connaissance déterminante de Patrice Coirault. Jusqu'à son entrée comme attaché de recherche au CNRS en 1955, c'est à titre privé qu'il mène ses enquêtes, même si, semble-t-il sur les conseils de l'abbé François Falc'hun (qui avait participé à la mission de folklore musical en Basse-Bretagne en 1939), il se décide à engager une thèse sur la tradition populaire de la danse en Basse-Bretagne, sous la direction d'André Leroi-Gourhan. Cela ne se fait toutefois pas sans difficultés, car le sujet n'intéresse guère les milieux de la recherche française. En 1958, à l'invitation de Georges-Henri Rivière, Jean-Michel Guilcher rejoint le Musée national des arts et traditions populaires pour y fonder un département de choréologie qui ne dispose en réalité d'aucun moyen financier. Dans le cadre d'un CNRS, qui lui laisse une forme de liberté, il peut poursuivre et mener à bien ses recherches et soutenir sa thèse (pour l'anecdote le président de son jury n'est autre qu'Henri-Irénée Marou) dont les résultats sont publiés en 1963 sous le titre La Tradition 
populaire de la danse en Basse-Bretagne ${ }^{2}$. Environ 2000 personnes interrogées dans trois cent soixante-quinze communes! Ouvrage essentiel pour la connaissance des danses traditionnelles en Basse-Bretagne, il livre aussi une réflexion fine et pertinente qui renouvelle largement les questions de transmission, d'évolution, d'emprunt, etc. en milieu traditionnel : très vite se pose à lui la question de savoir si ce qu'il a constaté en Basse-Bretagne peut aussi s'appliquer à d'autres régions. Tandis qu'en 1964 il consacre sa thèse complémentaire à « La contredanse française. Ses origines. Son évolution » qui, en 1969, nourrira La Contredanse et les renouvellements de la danse française, un ouvrage capital sur l'histoire de la danse, Jean-Michel Guilcher élargit ses recherches à d'autres milieux traditionnels, menant notamment en Berry, en Béarn, au pays basque, des enquêtes de terrains dont les résultats sont publiés entre $1965^{3}$ et $1984^{4}$. Autant de travaux qui, s'ils font aujourd'hui autorité, n'ont pas toujours fait l'unanimité au sein du monde français de la recherche, ce dont témoignent par exemple les dissensions apparues pendant la recherche collective en Aubrac (1964-1966) dont les résultats ne seront publiés qu'en $1975^{5}$.

Aussi, quand, le $1^{\text {er }}$ janvier 1968, le sociologue Jean Cuisenier succède à Georges-Henri Rivière à la tête du musée des ATP, Jean-Michel Guilcher décide de quitter le Centre d'ethnologie française pour Brest et le Centre de recherche bretonne et celtique, créé en 1969 par l'historien Yves le Gallo, dont il devient le directeur adjoint. Il entraîne dans ses bagages un autre ethnologue, spécialiste de littérature orale, Donatien Laurent, qu'il a rencontré dès 1957. En effet, dans les années 1950, Jean-Michel Guilcher intervient régulièrement dans des stages d'été destinés à former jeunes et enseignants à la langue et à la culture populaire bretonnes, tant au sein du mouvement catholique du Bleun-Brug (Fleur de bruyère) dirigé par l'abbé Falc'hun que de celui des instituteurs laïcs d' Ar Falz (La Faucille) animé par Pierre Hélias, René-Yves Creston, etc. Rien d'étonnant donc à ce que, une fois à Brest, JeanMichel Guilcher multiplie les conférences et interventions destinées à former des étudiants ou chercheurs et les inciter, à son exemple, à se lancer dans des enquêtes de terrain. Dès la rentrée universitaire de 1971, il dispense à titre bénévole un enseignement informel. En 1974, l'opportunité se présente de

2. Publié par Mouton, Paris-La Haye, 1963, réédité en 1976, l'ouvrage l'a été une nouvelle fois en 1995 par les maisons d'éditions Chasse-Marée-ArMen à Douarnenez et Coop-Breizh à Spézet, précédé d' « Un regard sur une recherche ancienne » par Jean-Michel Guilcher (p. I-XXXVIII).

3. Jean-Michel Guilcher, "Les formes anciennes de la danse en Berry ", Arts et traditions populaires, janvier-mars 1965 , p. 3-34.

4. Jean-Michel Guilcher, La Tradition de danse en Béarn et Pays Basque français, Paris, Maison des sciences de l'homme, 1984, $727 \mathrm{p}$.

5. Jean-Michel Guilcher, «Les danses de l'Aubrac », dans L'Aubrac, t. V, Ethnologie contemporaine. Paris, Centre national de la recherche scientifique, 1975, p. 298-361. 
créer un véritable enseignement d'ethnologie, grâce au soutien de l'historien Jacques Le Goff, alors président de la VI ${ }^{\mathrm{e}}$ Section de l'École pratique des hautes études et, en mai 1976, se créée à Brest le Centre d'ethnologie de la France, antenne de l'ÉHÉss, dont la direction est bien entendu confiée à JeanMichel Guilcher qui assure aussi une bonne partie de l'enseignement. Parmi les sujets abordés : les méthodes de recherche, les milieux traditionnels et la transmission, la littérature populaire de tradition orale avec un traitement particulier pour la poésie chantée (lecture commentée de l'œuvre de Patrice Coirault), dont on aura une idée à la lecture de La Chanson folklorique de langue française ${ }^{6}$. Il lira et commentera également le cours rédigé par MarieLouise Tenèze sur le conte populaire.

Une quarantaine d'étudiants, Bretons pour la plupart, suivent les séminaires où interviennent de nombreuses personnalités de l'ethnologie pour des interventions ponctuelles ou plus suivies, comme celles de Nicole Belmont. Ils engagent des thèses sur différents aspects de la culture tant matérielle qu'immatérielle qui reposent sur les enquêtes de terrain chères à Jean-Michel Guilcher. Malheureusement, en décembre 1979, atteint par la limite d'âge, il quitte l'Université qui, il faut bien l'avouer, n'a pas vraiment cherché à retenir celui dont les recherches n'étaient pas alors considérées à leur juste valeur.

Installé à Meudon, non loin de l'Observatoire de Meudon, lieu clé du Centre national de la recherche scientifique, institution au sein de laquelle il a œuvré pendant un quart de siècle, terminant sa carrière comme maître de recherche, il poursuit un enseignement informel et dispose surtout du temps nécessaire pour rédiger des ouvrages de synthèse... et ce temps, il le prend tant pour le contenu que pour la forme, car comme toujours chez lui, chaque mot est pesé, jusqu'à la ponctuation voire l'interlignage.

La genèse de Rondes, branles, caroles, le chant dans la danse qui paraît en $2003^{7}$ donne une belle idée de la personnalité du chercheur : à l'occasion du colloque international organisé à Quimperlé pour le centenaire de la naissance de Théodore Hersart de la Villemarqué, Donatien Laurent et moimême l'avions sollicité pour une intervention sur les liens entre le chant et de la danse. Il avait aimablement décliné l'invitation en arguant qu'il n'avait pas grand-chose d'intéressant à dire sur un sujet pour lequel il n'était guère compétent. Nous nous sommes permis d'insister... et il finit par accepter, «par amitié », nous dit-il. Non seulement son intervention fut, comme toujours, claire, précise et stimulante, mais il continua à travailler sur le sujet jusqu'à la rédaction de l'ouvrage précité. C'est encore, en 2009, Danse traditionnelle

6. Créteil, L'Atelier de la danse populaire, 1985, d'après des notes prises lors de ses cours.

7. Publié conjointement par le Centre de recherche bretonne et celtique et l'Atelier de la danse populaire. 
et anciens milieux ruraux français. Tradition, histoire, société ${ }^{8}$, un ouvrage de référence, synthèse capitale de plus d'un demi-siècle d'enquêtes et d'analyses en différentes régions françaises.

Malgré la qualité de travaux, pour lesquels Claude Lévi-Strauss se montre élogieux, la reconnaissance se fait attendre. C'est seulement en 1989 que ses amis du Centre alpin et rhodanien à Grenoble prennent l'initiative de rendre à Jean-Michel Guilcher un premier hommage bien mérité dont on trouvera les contributions rassemblées dans Tradition et histoire dans la culture populaire. Rencontres autour de l'œuvre de Jean-Michel Guilcher'. Il faut ensuite attendre 2014, et son centenaire, pour que, à Paris, puis à Brest, soit organisé un jubilé scientifique dont une partie des interventions sont reprises en 2015 dans un cahier spécial de la revue Ethnologie française ( $3^{\mathrm{e}}$ trimestre, $\mathrm{n}^{\circ} 153$ ).

Mais, pour connaître l'homme et l'œuvre, le mieux est peut-être de l'écouter. Le 3 avril 2014, il accorde à Jean-Luc Germain, journaliste à la revue Bretagne magazine histoire, un long entretien qu'il présente en quelque sorte comme sa dernière leçon. Cet enregistrement, où Jean-Michel Guilcher dresse le bilan d'une vie de recherche, a fait l'objet d'un don au CRBC par Bretagne magazine histoire. Il peut être consulté sur le site du CRBC à l'adresse suivante : purl.org/poi/crd. vjf.cnrs.fr/crdo-JAP_0001_SOUND et purl.org/ poi/crdo.vjf.cnrs.fr/crdo-JAP_0001_SOUND.

Qu'il soit permis à celui qui fut son étudiant et dont il dirigea les travaux de rendre hommage au remarquable chercheur et pédagogue qu'il fut et de témoigner sa profonde gratitude aux qualités humaines d'un maître auprès duquel il a tant appris.

FAÑCh Postic

Membre associé du CRBC

Université de Bretagne occidentale, Brest

8. Paris, L'Harmattan, 2009.

9. Grenoble, Centre alpin et rhodanien d'ethnologie, 1990. 